\title{
Exercise electrocardiography and aortic Doppler velocimetry in asymptomatic identical twins discordant for type 1 (insulin dependent) diabetes
}

S Dubrey, F Akhras, G J Song, T Hardman, C Travill, J Hynd, M I M Noble, S S S Lo, R D G Leslie

\begin{abstract}
Objective-To determine the influence of insulin dependent diabetes on the prevalence of myocardial ischaemia and on global left ventricular systolic performance.

Design-Stress treadmill electrocardiograms and simultaneous Doppler measurement of aortic maximum acceleration were obtained during exercise on symptom free subjects. The electrocardiograms were scored blindly according to the Minnesota code.
\end{abstract}

Participants-39 identical twin pairs (22 male) discordant for insulin dependent diabetes and 39 non-diabetic controls of similar age and sex were examined. The twins and controls had a mean age of 37 (range 25-69) with a mean (SD) duration of diabetes in the diabetic twin of 17 (7) years. Those selected were normotensive and had no renal impairment.

Results-Systolic blood pressure was significantly higher in the diabetic twins than in their non-diabetic cotwins both at rest $(p<0.05)$ and at peak exercise (p < 0.01). Electrocardiographic evidence of ischaemia was not correlated within twin pairs and was found in similar numbers of diabetic twins, their non-diabetic cotwins, and control subjects. Abnormal electrocardiograms were found in a similar number of diabetic twins (23\%), non-diabetic cotwins (18\%), and controls (15\%). There was a significant correlation in Doppler measurements of global left ventricular systolic function within the identical twins; no significant difference was found for these Doppler measurements in the diabetic twins, non-diabetic cotwins, or controls.

Conclusion-Exercise characteristics and cardiac function seem to be subject to shared genetic or shared environmental influences or both, whereas electrocardiographic features of ischaemia seem to be environmentally determined. In a selected cohort of diabetic identical twins without evidence of nephropathy there was no evidence that diabetes influenced the prevalence of myocardial ischaemia or global left ventricular systolic function.

(Br Heart f 1994;71:341-348)

Premature coronary artery disease is an important cause of morbidity and mortality in juvenile onset insulin dependent diabetes mellitus. ${ }^{1-2}$ The excess mortality from coronary artery disease is predominantly attributed to diabetic patients with persistent proteinuria, a hallmark of diabetic nephropathy. ${ }^{3} \mathrm{~A}$ less striking but considerably increased risk of coronary artery disease has also been reported in insulin dependent diabetes even in the absence of diabetic nephropathy. ${ }^{3-4}$ This effect could be due either to an influence of diabetes on risk factors for coronary artery disease or to an unrecognised genetic susceptibility to coronary disease. Risk factors for coronary artery disease are, however, only minimally altered by insulin dependent diabetes when renal function is normal and if diabetic control is good. ${ }^{5-6}$ To examine the effect of insulin dependent diabetes on the prevalence of myocardial ischaemia, in the absence of diabetic nephropathy, we have studied genetically identical twins discordant for diabetes. Myocardial ischaemia was estimated with exercise electrocardiography and global left ventricular systolic function with Doppler aortic velocimetry. Measurement of left ventricular outflow characteristics at the ascending aorta with Doppler ultrasound techniques allows an assessment of the global systolic function of the heart. ${ }^{7-8}$ Doppler aortic velocimetry has been shown to determine the presence $^{9-11}$ and extent of ischaemic left ventricular dysfunction during exercise. ${ }^{12}$ Twin studies with exercise electrocardiography and echocardiography have shown considerable heritability for both rest and exercise induced blood pressures ${ }^{13}$ and heart structure. ${ }^{14-16}$ The study of identical twins discordant for diabetes is a powerful method for estimating the influence of insulin dependent diabetes on a feature that is, in part, inherited. Identification of myocardial ischaemia in the non-diabetic identical twin of a patient with insulin dependent diabetes would establish whether any tendency to myocardial ischaemia was genetically determined.

\section{Patients and methods}

SUBJECTS

We recruited identical twins discordant for insulin dependent diabetes. For this study twin pairs were selected because they: $(a)$ were discordant for type 1 (insulin dependent) diabetes; $(b)$ were 25 to 69 years of age; (c) were of European origin; (d) had normal renal function; $(e)$ were taking no cardioactive medications; $(f)$ were not hypertensive; and $(g)$ both twins of a pair were available for study. 
Table 1 Clinical and laboratory characteristics of the diabetic twins, their non-diabetic cotwins, and the controls (results are means (SD) (median, range))

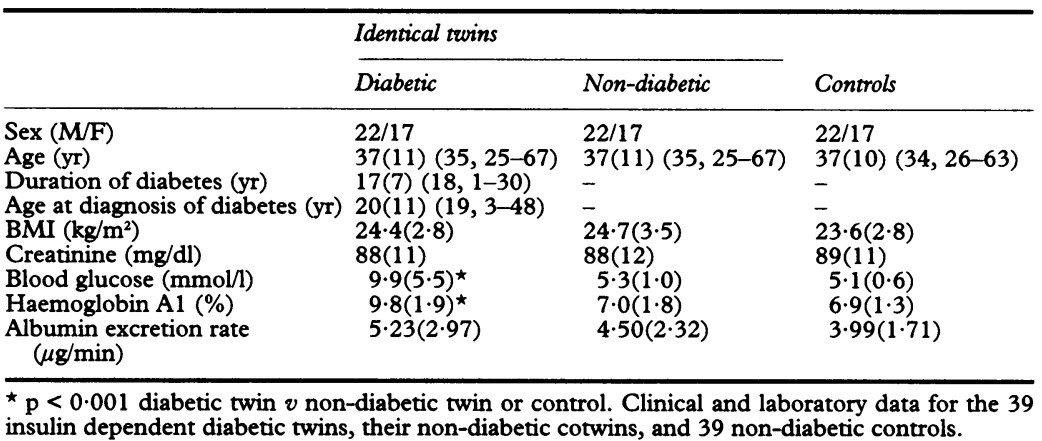

Hypertension was defined according to the World Health Organisation criterion (blood pressure exceeding $160 / 95 \mathrm{~mm} \mathrm{Hg}) .{ }^{17}$ Normal renal function was defined as showing no evidence of an increased rate of urinary albumin excretion on a timed overnight collection (albumin excretion rate $<30 \mu \mathrm{g} / \mathrm{min}$ ). Insulin dependent diabetes was defined according to the criteria of the National Diabetes Data Group. ${ }^{18}$ Of 53 identical twin pairs available for study, from the British Diabetic Twin Study Group, 39 fulfilled the above criteria. The diabetic twins had been treated from the time of diagnosis with insulin and currently took either highly purified porcine or human insulin.

The twins were compared with 39 nondiabetic control subjects, matched for age and sex; table 1 shows their clinical and laboratory characteristics. Control subjects were sought from unrelated members of the local community who were not attached to the hospital, had no family history of diabetes, were not taking medication, had normal renal function, were not hypertensive, and had no clinical signs or symptoms of illness.

None of the twin pairs or controls had a clinical diagnosis of ischaemic heart disease before the study and no subjects were excluded on these grounds. Subjects gave informed consent and the study was approved by the Hospital Ethics Committee.

\section{METHODS}

A full medical history was obtained from each subject, including details of work and leisure time physical activity, cigarette habits, and alcohol consumption. Information was requested regarding any family history of heart disease, diabetes, hyperlipidaemia, renal disease, or hypertension. Height and weight were measured with the subjects in light clothing without shoes to determine the body mass index.

\section{Exercise electrocardiography and Doppler measurements}

A 12 lead resting electrocardiogram was initially recorded, both with the patient supine and standing, immediately before starting the treadmill exercise test. All the identical twin pairs and the controls performed an exercise treadmill stress test (Bruce protocol) with simultaneous Doppler measurements of aortic blood flow at each stage of this exercise pro- tocol. Electrocardiograms were recorded at the end of each three minute exercise stage, at peak exercise, on immediate recovery, and every two minutes throughout a six minute recovery period. Signal averaged recordings as well as real time 12 lead records, running at a paper speed of $25 \mathrm{~mm} . \mathrm{s}^{-1}$, were acquired with a MAX-1 system Marquette apparatus (Marquette Inc, Milwaukee, WI USA). The exercise protocol was symptom limited but designed to stop on reaching an individual patient's maximum age predicted heart rate (defined as a heart rate of 220 minus the subjects age in years). The electrocardiograms were subjected to Minnesota coding ${ }^{19}$ in London by a blinded observer who had coded for the World Health Organisation multinational study of vascular disease in diabetic patients. Electrocardiograms were recorded as pathological and indicative of ischaemia according to the Whitehall criteria, defined as one or more of the following Minnesota code items: Q/QS waves (codes 1:1-1:3); ST segment depression (codes $4: 1-4: 3$ ); $\mathrm{T}$ waves inverted, diphasic, or flattened (codes $5: 1-5: 3$ ), or left bundle branch block (code 7:1:). ${ }^{20}$ Up sloping ST segment depression on exercise was regarded as abnormal when 2 $\mathrm{mV}$ of depression occurred at $80 \mathrm{~ms}$ after the $\mathrm{J}$ point. All other codings were regarded as normal.

The QT interval was measured from the electrocardiograms of all twins at rest and at each stage of the Bruce protocol, including at peak exercise. The QT interval values were plotted graphically against the heart rate for each individual identical twin pair and statistical analysis was used to compare the resultant regression lines.

Ascending aortic blood flow acceleration was determined transcutaneously from the suprasternal approach by means of a continuous wave Doppler blood velocity meter (Exerdop, Quinton Instrument, Seattle, Washington), transmitting ultrasound at a frequency of $3.0 \mathrm{MHz}$. This system is capable of measuring the maximum acceleration of blood flow in the range $2-99 \mathrm{~m} . \mathrm{s}^{-2}$, providing beat by beat digital output of these variables. The Doppler transducer was placed in the suprasternal notch and positioned to gain optimal signals by a combination of acoustic and visual feedback. The Exerdop provided mean values for maximum acceleration and peak velocity of aortic blood from at least 30 individual beats at each measurement point. Measurements were made at rest, two minutes into each stage of the exercise protocol, and immediately after the end of exercise. Doppler measurements were timed before the electrocardiographic recordings to exclude the possibility of interference with the electrocardiogram. In most cases (95\%) individual twin pairs were examined and tested at the same time of day, date, and location and with the same equipment.

\section{Blood pressure measurements}

Resting blood pressure was taken as the mean of three measurements of systolic and 
diastolic pressure from the right arm after the subject had been sitting for at least 10 minutes in a quiet setting. Readings were based on Korotkoff sounds I and V and taken five minutes apart by the same observer (SD) to the nearest $2 \mathrm{~mm} \mathrm{Hg}$, with a standard mercury sphygmomanometer and the appropriately sized arm cuff. The identical twins and their controls performed maximal exercise stress tests allowing blood pressures to be recorded under exercise conditions. Systolic and diastolic blood pressure measurements were made two minutes into each exercise stage of the protocol and as near to peak exercise duration as practical to obtain a peak exercise related blood pressure.

\section{Biochemical analysis and confirmation of monozygosity}

Random whole blood glucose was measured at the time of testing to exclude diabetes; control subjects with glucose concentrations $>6.7 \mathrm{mmol} / \mathrm{l}$ were excluded. Blood glucose was measured by a glucose oxidase method (Yellow Springs Instruments). Glycosylated haemoglobin was measured by electroendosmosis with the Corning method. Diabetes was excluded in all the non-diabetic cotwins by a $75 \mathrm{~g}$ oral glucose tolerance test and by a random whole blood glucose test at the time of testing. Normal blood glucose concentrations were defined as whole blood glucose $<5.6 \mathrm{mmol} / 1$ when fasting and $<6.7 \mathrm{mmol} / \mathrm{l}$ two hours after the oral glucose load. Blood samples were taken by venepuncture for biochemical analysis and to establish zygosity of the twin pairs. Monozygosity was determined for all twin pairs by history and serological examination of 23 blood groups including $\mathrm{ABO}$, rhesus (subgroups), Lewis, MNSs, Kidd, Kell, and Duffy and determination of the important histocompatability human leucocyte antigens (A, B, C, and DR loci). Discordance in one genetic system was sufficient to classify the twins as dizygotic and to exclude them from this study.

\section{Statistical analysis}

The null hypothesis within identical twin pairs was examined with a paired Student's $t$ test. Identical twin pairs were compared with their unrelated control subjects with an unpaired Student's $t$ test. Data that were not normally

Table 2 Exercise and haemodynamic data at rest and at peak exercise for the identical twins and their controls

\begin{tabular}{|c|c|c|c|}
\hline & \multicolumn{2}{|c|}{ Identical twins } & \multirow[b]{2}{*}{$\begin{array}{l}\text { Controls } \\
(n=39)\end{array}$} \\
\hline & $\begin{array}{l}\text { Diabetic } \\
(n=39)\end{array}$ & $\begin{array}{l}\text { Non-diabetic } \\
(n=39)\end{array}$ & \\
\hline $\begin{array}{l}\text { Duration of exercise (s) } \\
\text { Resting heart rate (beats/min) } \\
\text { Maximum heart rate (beats/min) } \\
\text { Predicted heart rate (\%) } \\
\text { Time to predicted heart rate (s) } \\
\text { Resting systolic BP (mm Hg) } \\
\text { Resting diastolic BP (mm Hg) } \\
\text { Maximum systolic BP }(\mathrm{mm} \mathrm{Hg}) \\
\text { Maximum diastolic BP (mm Hg) } \\
\text { Peak rate-pressure product } \\
\left.\text { (beat.min }{ }^{-1} \cdot \text { mm } \mathrm{Hg}^{-10^{-2}}\right)\end{array}$ & $\begin{array}{c}701(186) \\
89(13) \\
179(11) \\
97 \\
563(199) \\
127(17)^{\star} \\
79(10) \\
180(24)^{\star} \\
74(14) \\
316(46)^{\star}\end{array}$ & $\begin{array}{l}704(186) \\
87(13) \\
180(12) \\
98 \\
571(189) \\
123(18) \\
78(10) \\
170(24) \\
76(12) \\
302(44)\end{array}$ & $\begin{array}{l}764(201) \\
81(12) \\
180(11) \\
98 \\
641(167) \\
122(14) \\
81(10) \\
166(21) \\
74(12) \\
299(39)\end{array}$ \\
\hline
\end{tabular}

$\star p<0.05$ diabetic $v$ non-diabetic twin; ${ }^{\star \star} p<0.01$ diabetic twin $v$ non-diabetic twin and control. Results are expressed as mean (SD). distributed were analysed with the Wilcoxon matched pairs test. Similarities within twin pairs were assessed with the Pearson product moment correlation. A $\chi^{2}$ test was used to assess the significance of similarities for electrocardiographic features within twin pairs. Pooled Doppler data were analysed by one way analysis of variance. Regression lines for electrocardiographic QT interval $v$ heart rate were compared within twin pairs by analysis of covariance. Results are expressed as means (SD) and the median and range are included when values were not normally distributed. Values for $\mathrm{p}<0.05$ were regarded as significant.

\section{Results}

Mean (SD) random blood glucose and glycated haemoglobin were significantly higher in the diabetic twins $(9.9(5.5) \mathrm{mmol}$ and $9.8 \%(1.9 \%)$ than in the non-diabetic cotwins $(5.3(1.0) \mathrm{mmol}$ and $7.0 \%(1.8 \%)$, p $<0.001)$, and the matched controls $(5 \cdot 1$ $(0.6) \mathrm{mmol}$ and $6.9 \%(1.3 \%) \mathrm{p}<0.001)$. The overall level of glycaemic control in the diabetic twins indicated reasonable diabetic management; that for the non-diabetic twins and the control group are within the non-diabetic range for our laboratory $(5 \cdot 5 \%-7 \cdot 6 \%)$. Random blood glucose and glycated haemoglobin values were similar between the nondiabetic cotwins and the matched control group (table 1).

HEART RATE AND HAEMODYNAMICS AT REST AND ON EXERCISE

Within the identical twin pairs there was a significant correlation for heart rate at rest $(r=$ $0.39, \mathrm{p}<0.05)$. Mean resting heart rates were similar in the diabetic twins (89 (13) beats/min), their non-diabetic cotwins ( 87 (13) beats/min), and controls (81 (12) beats/min). Within the identical twin pairs, there was a significant correlation for both systolic $(r=0.77, \mathrm{p}<0.001)$ and diastolic $(r=0.59, \mathrm{p}<0.001)$ blood pressures at rest. The systolic blood pressure at rest was significantly higher in the diabetic twins (127 (17) $\mathrm{mm} \mathrm{Hg}$ ) compared with the non-diabetic cotwins (123 (18) $\mathrm{mm} \mathrm{Hg}, \mathrm{p}=0.034)$, but was similar between the non-diabetic twins and controls (122 (14) $\mathrm{mm} \mathrm{Hg}$ ). Diastolic blood pressures at rest did not differ significantly between the diabetic twins (79 (10) $\mathrm{mm} \mathrm{Hg}$ ), their non-diabetic cotwins (78 (10) $\mathrm{mm} \mathrm{Hg}$ ), or the controls $(81$ (10) $\mathrm{mm} \mathrm{Hg}$, (table 2).

Both the duration of exercise and the percentage of an age predicted heart rate reached on exercise were significantly correlated $(r=$ $0.81, \mathrm{p}<0.0001$ and $r=0.51, \mathrm{p}<0.001)$; mean values were similar between the diabetic twins (701 s and 97\%), their non-diabetic cotwins (704 s and $98 \%$ ), and the controls (764 s and 98\%). The mean time to reach $90 \%$ of the age predicted heart rate was similar between the diabetic twins (563 (199) s), the non-diabetic cotwins (571 (189), s) and the controls (641 (167) s). 
Table 3 Electrocardiographic features in the identical twins and controls

\begin{tabular}{|c|c|c|c|c|}
\hline \multirow{2}{*}{$\begin{array}{l}\text { Electrocardiographic } \\
\text { feature (Minnesota } \\
\text { code) }\end{array}$} & \multicolumn{2}{|c|}{ Identical twins } & \multirow{2}{*}{$\begin{array}{l}\text { Pairs } \\
\text { concordant } \\
\text { for feature } \\
(n)\end{array}$} & \multirow[b]{2}{*}{ Controls } \\
\hline & Diabetic & Non-diabetic & & \\
\hline \multicolumn{5}{|l|}{ ST depression: } \\
\hline Major $(4: 1)$ & 0 & 0 & 0 & 0 \\
\hline Intermediate $(4: 2)$ & 2 & 2 & 1 & 0 \\
\hline Minor $(4: 3)$ & 2 & 1 & 0 & 0 \\
\hline Up sloping (4:4) & 6 & 7 & 1 & 10 \\
\hline Total $(4: 1-4: 4)$ & 10 & 10 & 2 & 10 \\
\hline ST elevation $(9: 2)$ & 0 & 0 & 0 & 2 \\
\hline \multicolumn{5}{|l|}{$\mathrm{T}$ wave inversion: } \\
\hline Major $(5: 1)$ & 0 & 0 & 0 & 1 \\
\hline Intermediate $(5: 2)$ & 5 & 3 & 1 & 1 \\
\hline Minor (5:3) & 4 & 4 & 1 & 5 \\
\hline Total $(5: 1-5: 3)$ & 9 & 7 & 3 & 6 \\
\hline \multicolumn{5}{|l|}{ AV conduction defect: } \\
\hline Short PR (6:5) & 6 & 2 & 2 & 2 \\
\hline \multicolumn{5}{|l|}{ Ventricular conduction defect: } \\
\hline Left BBB $(7: 1: 1)$ & 1 & 0 & 0 & 0 \\
\hline Right BBB (7:2:1) & 0 & 1 & 0 & 0 \\
\hline Total $(7: 1: 1-7: 2: 1)$ & 1 & 1 & 1 & 0 \\
\hline \multicolumn{5}{|l|}{ Rhythm and rate: } \\
\hline $\begin{array}{l}\text { Frequent ventricular } \\
\text { extrasystoles }(8: 1: 2)\end{array}$ & 2 & 0 & 0 & 0 \\
\hline $\begin{array}{l}\text { Frequent atrial } \\
\text { extrasystoles }(8: 1: 1)\end{array}$ & 0 & 0 & 0 & 1 \\
\hline $\begin{array}{l}\text { Isolated extrasystoles }<10 \% \\
\text { Sinus rate }>100 \text { at rest }(8: 7)\end{array}$ & $\begin{array}{l}5 \\
8\end{array}$ & $\begin{array}{l}3 \\
6\end{array}$ & $\begin{array}{l}2 \\
1\end{array}$ & $\begin{array}{r}11 \\
5\end{array}$ \\
\hline $\begin{array}{l}\text { Pathological electrocardiographic } \\
\text { features (n) }\end{array}$ & 14 & 10 & 4 & 6 \\
\hline $\begin{array}{l}\text { Subjects with pathological } \\
\text { electrocardiograms }(\mathrm{n}(\%))\end{array}$ & $9(23)$ & $7(18)$ & 2 & $6(15)$ \\
\hline
\end{tabular}

Pathological electrocardiograms are defined according to the Whitehall criteria for electrocardiographic evidence of myocardial ischaemia; including Minnesota codes for $Q$ or QS waves (codes (codes 5:1-5:3) or left bundle branch block (code $7: 1:) .^{20}$

Within the identical twin pairs there was a significant correlation for heart rate at peak exercise $(r=0.80, \mathrm{p}<0.0001)$. At peak exercise the maximum heart rate did not differ within the identical twin pairs or between either twin and control (table 2). Within the identical twin pairs, there was a significant correlation for both peak systolic $(r=0.66$, p $<0.001)$ and diastolic $(r=0.59, \mathrm{p}<0.001)$ blood pressures. The maximum recorded systolic blood pressure was significantly higher in the diabetic twin $(180(24) \mathrm{mm} \mathrm{Hg})$ ) compared with the non-diabetic cotwins (170 (24) $\mathrm{mm} \mathrm{Hg}, \mathrm{p}=0.003$ ) and the controls (166 (21) $\mathrm{mm} \mathrm{Hg}, \mathrm{p}=0.009$ ). Maximum diastolic blood pressure recorded on exercise was similar in the diabetic twin (74 (14) $\mathrm{mm}$ $\mathrm{Hg}$ ), the non-diabetic cotwin (76 (12) $\mathrm{mm}$ $\mathrm{Hg}$ ), and controls (74 (12) $\mathrm{mm} \mathrm{Hg})$. The resultant peak rate-pressure product (mean (SD), beat. $\mathrm{min}^{-1} \mathrm{~mm} \mathrm{Hg} .10^{-2}$ ) was significantly higher in the diabetic twins $(316(46))$ than in the non-diabetic cotwins (302 (44), p = $0.026)$; but did not differ between either twin and the controls (299 (39)).

\section{ELECTROCARDIOGRAPHIC FINDINGS}

(MINNESOTA CODE NUMBERS)

None of the identical twins or controls experienced chest discomfort compatible with myocardial ischaemia during the exercise stress test or recovery period. Rhythm disturbances of haemodynamic significance did not occur throughout this study. Table 3 shows the prevalence of the more frequent individual Minnesota coded electrocardiographic findings (code numbers), occurring at rest and on exercise, in the identical twin pairs and controls. Resting electrocardiograms were often morphologically similar within individual twin pairs, whereas on exercise there was often a discordance for electrocardiographic changes (fig 1 and 2). None of the identical twins or controls showed $Q$ waves (codes 1:1-1:3) indicative of previous myocardial infarction.

Electrocardiographic ST segment depression (codes $4: 2-4: 4)$ was found in nine $(23 \%)$ diabetic twins (six were up sloping (code 4:4), nine $(23 \%)$ non-diabetic twins (seven were up sloping (code $4: 4)$, and in $10(26 \%)$ of the controls (all 10 were up sloping (code 4:4)). Of 18 twins with ST segment depression, only two pairs were concordant; the remaining seven diabetic and seven non-diabetic twins were discordant for ST segment depression.
Baseline

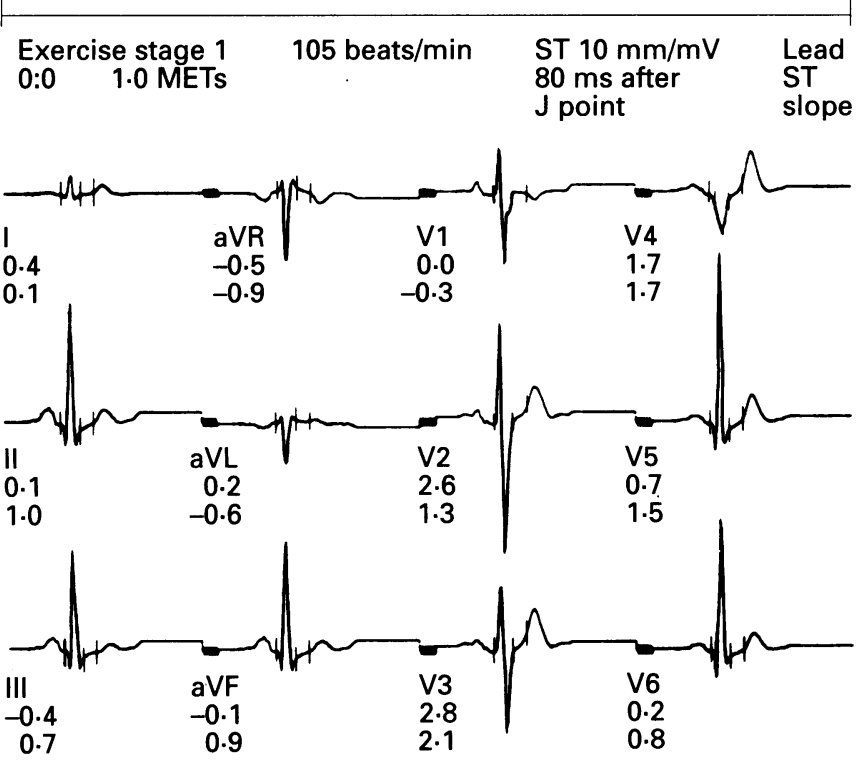

Max ST

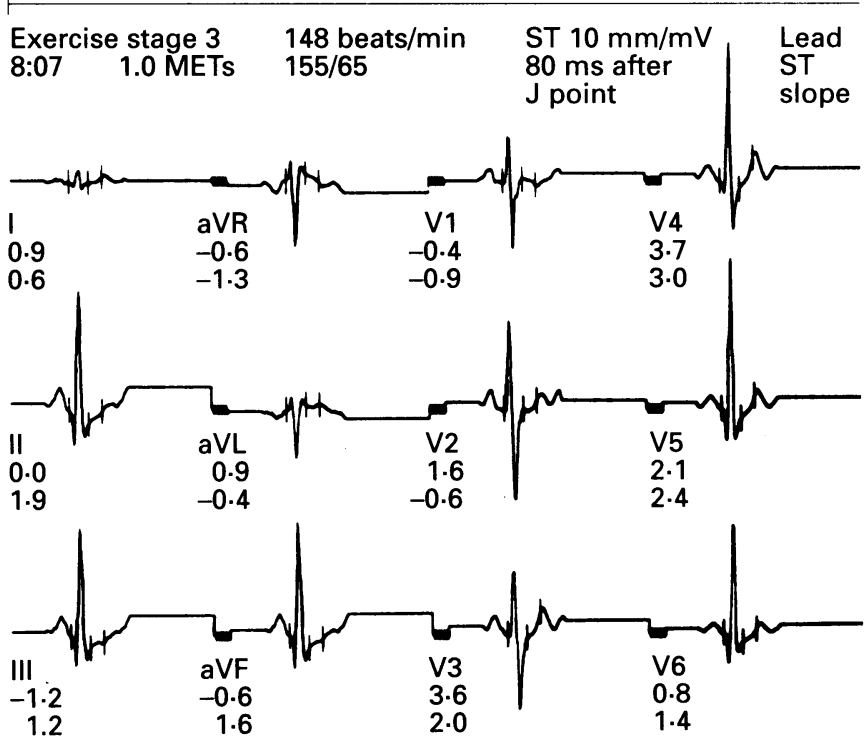
Figure 1 Electrocardiograms in a 51 year old male twin, diabetic for 30 years at the start of exercise (Baseline) and at the point of maximum $S T$
segment change on exercise (Max ST). The similarity of resting configuration and the differences on exercise can be appreciated compared with those of his identical non-diabetic cotwin in fig 2. 
Baseline

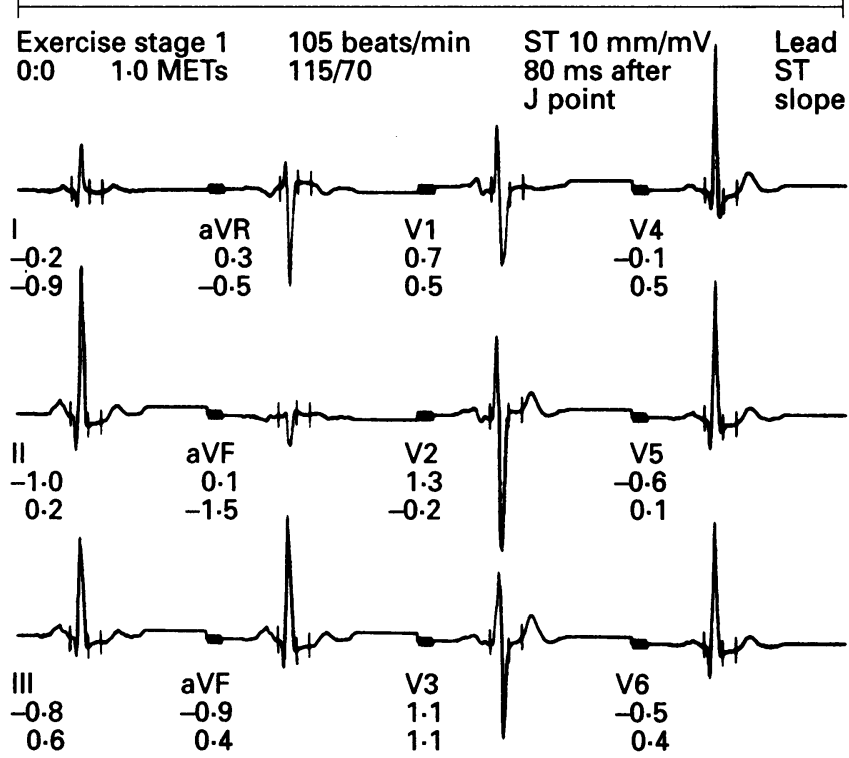

$\operatorname{Max}$ ST

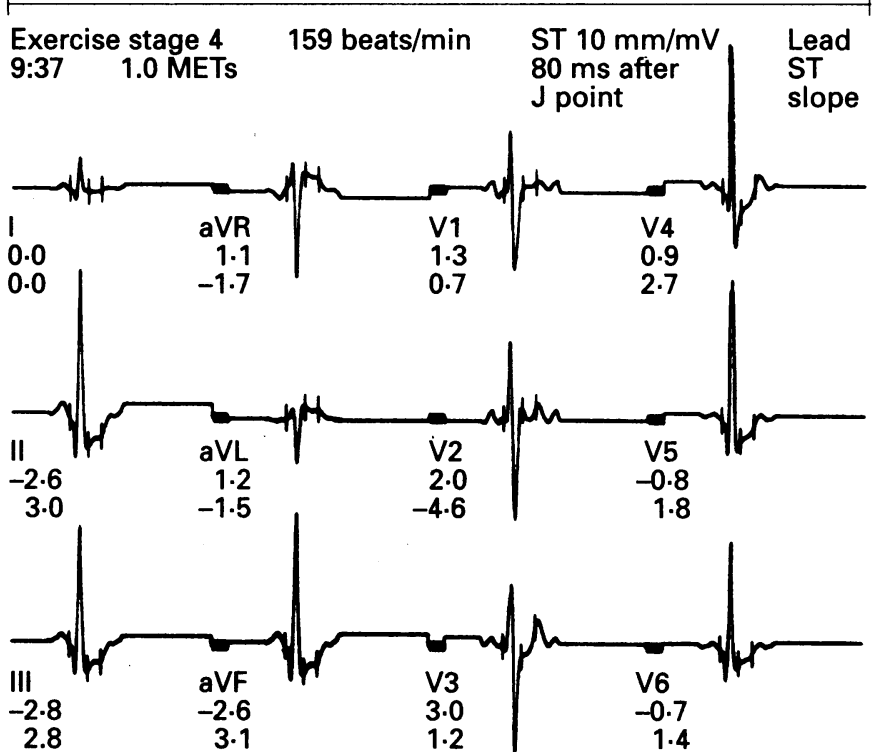

Figure 2 Electrocardiograms in a 51 year old male identical non-diabetic twin of subject in fig 1 at the start of exercise (Baseline) and at the point of maximum ST segment change on exercise (Max ST). The similarity of resting morphology and the differences on exercise can be appreciated compared with those of his identical diabetic cotwin in fig 1 .

No twins or controls were coded for major ST segment depression (code 4:1). Electrocardiographic ST segment elevation (code 9:2) was found in only two of the control subjects on exercise and in none of the twin subjects. The prevalence of $T$ wave inversion (codes 5:1-5:3) was similar within the identical twins and controls with eight $(21 \%)$ diabetic twins, seven (18\%) non-diabetic twins, and six $(15 \%)$ controls having major (code 5:1), intermediate (code $5: 2$ ), or minor (code $5: 3$ ) $T$ wave changes. Of 15 twins with $T$ wave inversion, only three pairs were concordant; the remaining five diabetic and four non-diabetic twins were discordant for $T$ wave inversion.

The prevalence of an accelerated atrioventricular conduction, in the form of a short PR interval (code 6:5), was similar with six diabetic twins (15\%), two non-diabetic twins $(5 \%)$, and two controls $(5 \%)$ showing this feature at rest. Of eight twins with a short PR interval, only two pairs were concordant; four diabetic twins were discordant for a short PR interval.

Ventricular conduction defects were found in only two twin subjects of one identical pair, the diabetic twin having a left bundle branch block pattern (code 7:1:1) and the nondiabetic twin a right bundle branch block pattern (code $7: 2: 1$ ). No control subjects exhibited the features of a ventricular conduction defect.

Frequent premature ventricular extrasystoles (code $8: 1: 2$ ) were documented in only two twins ( $5 \%)$, both diabetic; isolated less frequent ventricular extrasystoles occurred in a similar number of diabetic (13\%) and nondiabetic twins $(8 \%)$ but in significantly more of the control subjects $(28 \%)$ than the nondiabetic twins $(p<0.05)$. The number of subjects having a resting sinus tachycardia (code 8:7), recorded in the immediate anticipation of performing an exercise test, was similar in the diabetic twins $(21 \%)$, non-diabetic twins (15\%), and controls (13\%).

Pathological electrocardiograms (Whitehall criteria) were documented in a similar number of diabetic twins (23\%), non-diabetic twins $(18 \%)$, and controls (15\%). Of 16 twins with pathological electrocardiographic findings only three twin pairs were concordant; the remaining six diabetic and four non-diabetic twins were discordant for these pathological electrocardiographic features. In the subjects who had pathological electrocardiograms there was no significant difference between the mean ages of the diabetic twins (43(15)), non-diabetic twins (38(8)), or the controls (32(5)). Also, the mean (SD) duration of diabetes in the diabetic twins (19)(8) years) with pathological electrocardiographic changes was similar to that of diabetic twins with normal electrocardiographic responses to exercise (15(7)y).

Of nine twin subjects showing both ST segment changes (codes 4:1-4:4) and $T$ wave inversion (codes 5:1-5:3); two identical pairs were concordant, whereas four diabetic and one non-diabetic twin were discordant for having these combined ST segment and T wave changes on exercise.

The QT interval shortened in all diabetic and non-diabetic twins as the heart rate increased with exercise. In 32 of the 39 pairs $(82 \%)$ there was no significant difference in the degree of shortening of the QT interval on increasing heart rate with exercise within each pair of twins. In the remaining seven twin pairs shortening of the QT interval with increasing heart rate was significantly greater in the diabetic twin in four pairs and in the non-diabetic twin in three pairs, relative to their respective cotwins $(p<0.05)$. 


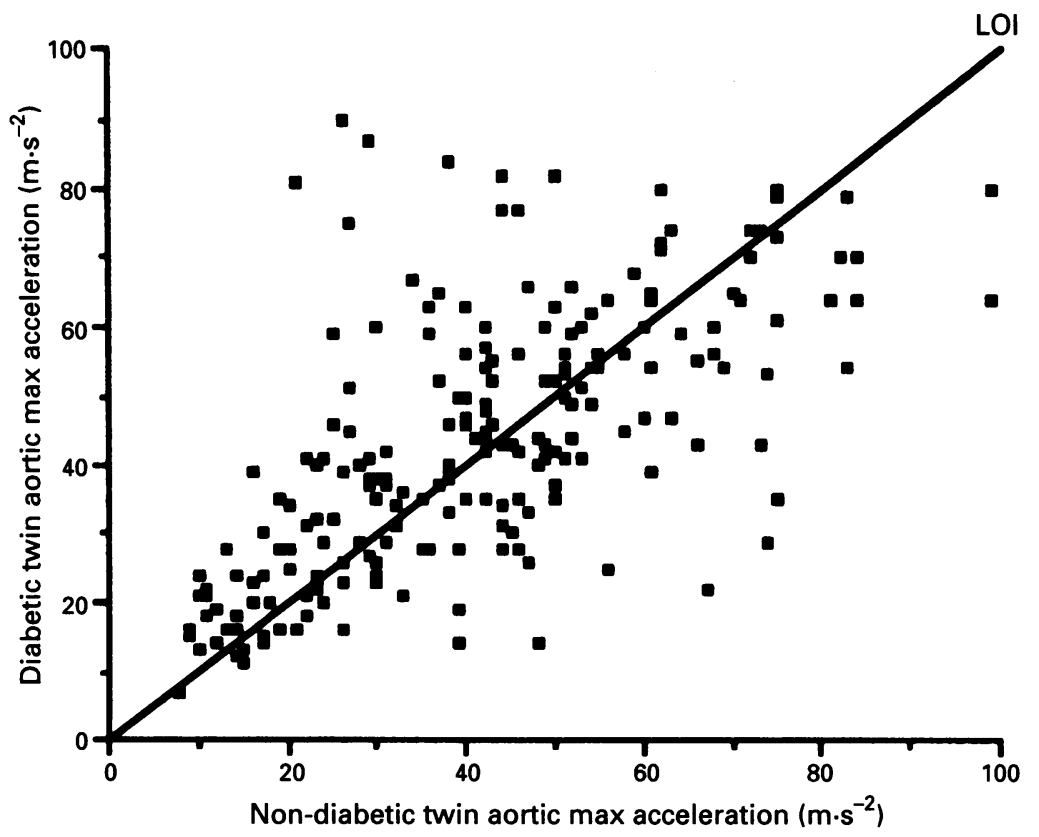

Figure 3 Cumulative mean Doppler aortic blood maximum acceleration values for the diabetic twins plotted against those for their non-diabetic cotwins throughout the exercise stress test. The bold line (LOI) represents the line of identity. The data points are significantly correlated $(r=0.61, p<0.001)$.

\section{DOPPLER DERIVED MEASUREMENTS OF LEFT}

VENTRICULAR SYSTOLIC PERFORMANCE

Doppler measurements of the maximum ascending aortic blood acceleration reached on exercise were significantly correlated within the twin pairs $(r=0.49, \mathrm{p}<0.001)$; but not between the controls and either the non-diabetic $(r=0 \cdot 22)$ or the diabetic twins $(r=0 \cdot 11)$. Figure 3 shows the individual mean values obtained, for all Doppler measurements of aortic acceleration, at rest and throughout the exercise phase of the protocol in the diabetic twins and their non-diabetic cotwins. The mean peak values for maximum aortic blood flow acceleration recorded on exercise were similar between the diabetic (56(15) ${\left.\mathrm{m} . \mathrm{s}^{-2}\right)}$ and non-diabetic (54(17)

Table 4 Doppler measurements of the maximum mean aortic acceleration for the identical twins and their controls at each stage of the exercise treadmill stress test

\begin{tabular}{llll}
\hline & \multicolumn{2}{l}{ Identical twins } & \\
\cline { 2 - 3 } Stage of test $(n)$ & Diabetic & Non-diabetic & Controls \\
\hline Before test (standing)(39) & $24(16)$ & $22(14)$ & $23(11)$ \\
I (39) & $38(17)$ & $37(18)$ & $37(15)$ \\
II (39) & $43(16)$ & $41(17)$ & $43(16)$ \\
III (39) & $51(15)$ & $49(16)$ & $50(17)$ \\
IV (31) & $50(13)$ & $49(15)$ & $53(16)$ \\
Recovery (39) & $53(17)$ & $51(18)$ & $47(16)$ \\
\hline
\end{tabular}

The values represent the mean (SD) aortic ascending blood flow acceleration $\left(\mathrm{m} . \mathrm{s}^{-2}\right)$.

Table 5 Doppler measurements of mean change (\%) in maximum aortic acceleration values from resting values at each stage of the exercise treadmill stress test

\begin{tabular}{lccc}
\hline & \multicolumn{2}{l}{ Identical twins } & \\
\cline { 2 - 3 } Stage test $(n)$ & Diabetic & Non-diabetic & Controls \\
\hline I (39) & $86(90)$ & $93(103)$ & $87(82)$ \\
II (39) & $122(93)$ & $127(122)$ & $117(104)$ \\
III (39) & $158(96)$ & $173(128)$ & $152(108)$ \\
IV (31) & $164(83)$ & $194(153)$ & $157(99)$ \\
Recovery (39) & $176(146)$ & $181(129)$ & $127(94)$ \\
\hline
\end{tabular}

The values represent the percentage increase in acceleration from that measured at baseline mean (SD)). $\left.\mathrm{m} . \mathrm{s}^{-2}\right)$ twin or the controls $\left(53(17) \mathrm{m} . \mathrm{s}^{-2}\right)$ Doppler measurements of maximum ascending aortic blood acceleration obtained at rest, within each individual stage of the Bruce protocol exercise test, or on recovery were not significantly different between the diabetic twins, the non-diabetic cotwins, or the controls (table 4). Also the percentage change in acceleration of aortic blood flow from rest to values obtained on exercise was similar between the diabetic twins, the non-diabetic cotwins and the controls (table 5).

\section{Discussion}

A study of identical twins discordant for insulin dependent diabetes provides a powerful method for examination of both the influence of diabetes and the genetic susceptibility to diabetes on the prevalence of myocardial ischaemia. In this selected cohort of patients, who were normotensive, normoalbuminuric, and had reasonable diabetic management, neither diabetes in itself nor a genetic susceptibility to insulin dependent diabetes conferred an increased risk of myocardial ischaemia or global systolic left ventricular dysfunction.

Twin studies have shown that both systolic and diastolic blood pressures are under considerable genetic influences both at rest and during stress conditions. ${ }^{1321}$ Our findings are consistent with such a genetic influence as there was a strong correlation for blood pressure between the twins we studied both at rest and on exercise. We previously found that identical twins with insulin dependent diabetes had a higher resting systolic blood pressure than their non-diabetic cotwins, ${ }^{22}$ a finding confirmed in this study. Also, we found that there was a disproportionate increase in systolic blood pressure in the diabetic twin during exercise. This exaggerated blood pressure response to exercise is a characteristic feature of patients with insulin dependent diabetes and incipient nephropathy, ${ }^{23-25}$ but also of patients with insulin dependent diabetes who are normoalbuminuric. $^{26-27}$ The exaggerated blood pressure response has been ascribed to a decreased urinary sodium excretion..$^{28}$ It seems to be secondary to diabetes as the non-diabetic twins had a normal blood pressure response to exercise.

Exercise electrocardiography is more accurate than radioisotopic tomographic imaging in the detection of asymptomatic coronary artery disease in diabetic patients. ${ }^{29}$ We therefore used exercise electrocardiography to test the hypothesis that patients with insulin dependent diabetes but without renal disease were more likely to show evidence of coronary artery disease. In agreement with previous studies we found a high prevalence of abnormal electrocardiograms in our diabetic twin subjects performing an exercise stress test. ${ }^{30-31}$ A similar rate of pathological electrocardiographic change was found within the identical twins, but there was also no significant difference between the number of non-diabetic and control subjects with electrocardiographic changes. The similarity in the frequency of 
electrocardiographic changes between the non-diabetic twins who retain the genetic susceptibility to develop diabetes, and the nondiabetic controls suggests that there is no significant influence from a genetic susceptibility to diabetes. Thus the hypothesis that these selected patients with insulin dependent diabetes would have a higher rate of coronary artery disease is not confirmed. An initial power analysis assuming a fourfold excess of electrocardiographic changes in the diabetic twins suggested that we should have a result approaching significance with the number of twins we studied. The frequency of ischaemic heart disease in this group at this age was unlikely to be that high but the number of electrocardiographic changes found was consistent with our initial power analysis. Thus there was a sufficient frequency of electrocardiographic change to confirm no significant difference between the diabetic and the nondiabetic twins. Also, it is important to note that the mean (SD) duration of insulin dependent diabetes was relatively long at 17 (7) years. Although the number of twins we studied was small and we cannot exclude a false negative result we would have to study a substantially greater number of twin pairs to identify any significant change, if indeed there is one. Given the $5 \%$ difference we have shown in the number of pathological electrocardiograms between the diabetic and the non-diabetic twins a power analysis shows that at a $90 \%$ confidence interval $(90 \% \mathrm{CI})$ we would need to recruit 1408 twin pairs to show a significant effect of diabetes. This is clearly not possible as it far exceeds the number of such twin pairs in the United Kingdom. Most importantly these findings emphasise that the effect, if any, of insulin dependent diabetes on ischaemic electrocardiographic changes must be small. There is currently some doubt whether there is an increased prevalence of silent ischaemia on exercise tests in asymptomatic patients with insulin dependent diabetes ${ }^{32}$; some studies reporting ischaemic changes to be no more common in diabetic than non-diabetic patients. ${ }^{33-35}$ It is likely, therefore, given our findings that either diabetes in the absence of renal disease is not associated with an excess risk of coronary artery disease or, alternatively, that this risk is extremely small.

Twin studies with exercise electrocardiography have shown that the heart rate is under significant genetic influence. ${ }^{36-38}$ Our findings confirmed the strong correlation between identical twins for heart rate, both at rest and on exercise, consistent with an important genetic influence. Similarities between identical twins for electrocardiograms are well established. ${ }^{39}$ We found that the identical twins were strikingly different for a range of pathological changes on their electrocardiograms. These differences between identical twins must be non-genetically determined. Whatever the relevant non-genetic factor or factors might be it is not diabetes as the changes occurred independently of the presence of insulin dependent diabetes.
An alternative contribution to the increased mortality in subjects with insulin dependent diabetes is that related to sudden cardiac death due to pathological disturbances of ventricular rhythm. Interest in the QT interval derives from reports suggesting that a prolonged QT interval may be a marker for an increased risk of sudden death in patients with ischaemic heart disease $e^{40-41}$ or diabetic autonomic neuropathy. ${ }^{42-44}$ We found no association between QT interval and age, duration of diabetes, degree of glycaemic control, or electrocardiographic evidence of myocardial ischaemia.

Whereas atrioventricular conduction time (PR interval) is reported to be under a strong genetic influence, ${ }^{3645}$ of the eight twins that showed a short PR interval only two twin pairs were concordant for this electrocardiographic feature. Ventricular conduction defects seem to be subject to genetic or shared environmental influences being concordant within one of the identical male twin pairs; the diabetic exhibiting the more sinister pattern of left bundle branch block and the non-diabetic cotwin that of right bundle branch block.

Twin studies have shown a significant genetic component to left ventricular structural features ${ }^{15}$ and aortic outflow characteristics. ${ }^{143746}$ Insulin dependent diabetes could influence global left ventricular function during exercise through ischaemia or a specific non-ischaemic diabetic cardiomyopathy. We found no evidence of impaired left ventricular contractility in the diabetic twins; the Doppler acquired measurements of ascending aortic blood flow, which reflect global left ventricular systolic performance, did not support the existence of either an excess of myocardial ischaemia or a specific cardiomyopathy in the diabetic twins.

In summary, exercise characteristics and cardiac function are subject to shared genetic and environmental influences. In our cohort electrocardiographic changes consistent with myocardial ischaemia were largely determined by non-genetic factors that did not include diabetes. Insulin dependent diabetes in the absence of renal disease may not be associated with a substantial excess risk of coronary artery disease.

SD was supported by a British Heart Foundation fellowship award. The study was supported by grants from the Wellcome Trust, the Diabetic Twin Research Trust, and the British Trust, the Diabetic Twin Research Trust, and the British
Diabetic Association. We acknowledge the help and advice of Diabetic Association. We acknowledge the help and advice of
Mrs N Keen in the interpretation of all electrocardiograms by Mrs N Keen in the interpretation of all electrocardiograms by
Minnesota coding. This material was presented at a plenary session of the Medical Research Society, 1993.

1 Krolewski AS, Kosinski EJ, Warram JH, Leland SO, Busick EJ, Asmal AC, et al. Magnitude and determinants of coronary artery disease in juvenile-onset insulindependent diabetes mellitus. Am $f$ Cardiol 1987;59. 750-5.

2 Valsania P, Zarich SW, Kowalchuk GL, Kosinski E, Warram JH, Krolewski AS. Severity of coronary artery disease in young patients with insulin dependent diabetes mellitus. Am Heart $\mathcal{f}$ 1991;122:695-700.

3 Jensen T, Borch-Johnsen K, Kofoed-Enevoldsen A Deckert $T$. Coronary heart disease in young type 1 (insulin dependent) diabetic patients with and without diabetic nephropathy: incidence and risk factors. Diabetologia 1987;30:144-8.

4 Borch-Johnsen K, Kragh Andersen P, Deckert T. The effect of proteinuria on relative mortality in type 1 
(insulin-dependent) diabetes mellitus. Diabetologia 1985; 28:590-6.

5 Pyorala K. Diabetes and coronary artery disease: what a coincidence? f Cardiovasc Pharmacol 1991;16(supp 9): 8-14.

6 Dubrey SW, Reaveley DA, Leslie RDG, O'Donnell M, O'Connor BM, Seed M. Effect of insulin dependent diabetes on lipids and lipoproteins: a study of identical twins. Clin Sci 1993;84:537-42.

7 Bennett ED, Barclay SA, Davis AL, Mannering D, Mehta N. Ascending aortic blood velocity and acceleration using Doppler ultrasound in the assessment of left ventricular dysfunction. Cardiovasc Res 1984;18:632-8.

8 Sabbah HN, Khaja F, Brymer JF, McFarland TM, Albert $\mathrm{DE}$, Snyder JE, et al. Noninvasive evaluation of left ventricular performance based on peak aortic blood acceleration measured with a continuous wave Doppler velocity meter. Circulation 1986;74:323-9.

9 Bryg RJ, Labowitz AJ, Mehdirad AK, Williams GA, Chaitman BR. Effect of coronary artery disease on Doppler-derived parameters of aortic flow during upright exercise. $A m \mathcal{F}$ Cardiol 1986;58:14-9.

10 Teague SM, Mark DB, Radford M, Rogertson J, Albert D, Waugh RA. Doppler ejection dynamics during ischemic exercise responses. Circulation 1985;72(111),448.

11 Cucchini F, DiMario C, Iavernaro A, Zeppellini R, Barilla A, Bolognesi R. Peak aortic blood acceleration: a possible indicator of initial left ventricular impairment in potients with coronary artery disease. Eur Heart $\mathcal{f} 1991$; patients with

12 Sabbah HN, Przybylski J, Albert DE, Stein PD. Peak aortic blood acceleration reflects the extent of left ventricular blood acceleration reflects the extent of left ventric

13 Hunt SC, Hasstedt SJ, Kuida H, Stults BM, Hopkins PM, Williams RR. Genetic heritability and common environmental components of resting and stressed blood pressures, lipids, and body mass index in Utah pedigrees and twins. Am $\mathcal{F}$ Epidemiol 1988;129(3):625-38.

14 Adams TD, Yanowitz FG, Fisher AG, Ridges JD, Nelson $\mathrm{AG}$, Hagan $\mathrm{AD}$, et al. Heritability of cardiac size: an echocardiographic and electrocardiographic study of monozygotic and dizygotic twins. Circulation 1985; 71:39-44.

15 Bielen E, Fagard A, Amery A. Inheritance of heart structure and physical exercise capacity: a study of left ventricular structure and exercise capacity in 7-year old twins. Eur Heart $₹$ 1990;11:7-16.

16 Verhaaren HA, Schieken RM, Mosteller M, Hewitt JK, Eaves LJ, Nance WE. Bivariate genetic analysis of left ventricular mass and weight in pubertal twins (The medical college of Virginia Twin Study). Am $₹$ Cardiol 1991;68:661-8.

17 World Health Organisation. Arterial hypertension. Report of a WHO expert committee on hypertension. Geneva: WHO 1979. (Tech rep series No 628.)

18 National Diabetes Data Group. Classification and diagnosis of diabetes mellitus and other categories of glucose intolina

19 Prineas RJ, Crow RS, Blackburn H. The Minnesota Code manual of electrocardiographic findings; standards and procedures for measurement and classification. Bristol: John Wright, 1982.

20 Rose G, Baxter PJ, Reid DD, McCartney P. Prevalence and prognosis of electrocardiographic findings in middle-aged men. Br Heart $f$ 1978; 40:636-43

21 Feinleib M, Garrison RJ, Fabsitz R, Christian JC, Hrubec Z, Borhani NO, et al. The NHLBI twin study of cardiovascular disease risk factors: methodology and summary of results. Am $\Im$ Epidemiol 1977;106:284-95.

22 Hardman TC, Dubrey SW, Leslie DG, Hafiz M, Noble MI, Lant AF. Erythrocyte sodium-lithium countertransport and blood pressure in identical twin pairs discordant for insulin dependent diabetes. BMF 1992;305: 215-9.

23 Carlstrom S, Karlefors T. Hemodynamic studies during exercise in newly diagnosed juvenile diabetics. Acta Me

Abnormal albuminuria and blood pressure rise in incipient diabetic nephropathy induced by exercise. Kidney Int 1984;25:819-23.
25 Garg SK, Chase HP, Jackson WE, Harris S, Marshall G, Hoops $S$. Retinal changes and alterations in blood pressure and albumin excretion rate (AER) during exercise in type 1 diabetes. Diabetes Res Clin Pract 1991;11:

26 Watts GF, Williams I, Morris RW, Mandalia S, Shaw KM, Polak A. An acceptable exercise test to study microalbuminuria in type 1 diabetes. Diabetic Med 1989; 6:787-92.

27 Chase HP, Garg SK, Harris S, Marshall G, Hoops S Elevation of resting and exercise blood pressure in subjects with type 1 diabetes and relation to albuminuria. 7 Diabetic Complications 1992;6:138-42.

28 Cavan D, O'Donnell MJ, Parkes A, Lewis H, Lawson N, Chapman JA, et al. Abnormal blood pressure response to exercise in normoalbuminuric insulin dependent diabetic patients. F Hum Hypertens 1991;5:21-6.

29 Koistinen MJ, Huikuri HV, Pirttiaho H, Linnaluoto MK, Takkunen JT. Evaluation of exercise electrocardiography and thallium tomographic imaging in detecting asymptomatic coronary artery disease in diabetic patients. Br Heart F 1990;63:7-11.

30 Storstein L, Jervell J. Response to bicycle exercise testing in long-standing

31 Levitas IM, Kristal J. Stress exercise testing of the young diabetic for the detection of unknown coronary artery disease. Isr ₹.Med Sci 1972;8:845-7.

32 Callaham PR, Froelicher VF, Klein J, Risch M, Dubach P, Friis R. Exercise induced silent ischaemia: age, diabetes Friis $R$. Exercise induced silent ischaemia: age, diabetes
mellitus, previous myocardial infarction, and prognosis. mellitus, previous myocardial infarction

33 Lindsey HE, Cohn PF. Silent myocardial ischaemia during and after exercise testing in patients with coronary artery disease. Am Heart 7 1978;95:441-7.

34 Smith JW, Buckels LJ, Carlson K, Marcus FI. Clinical characteristics and results of non-invasive tests in 60 diabetic patients after myocardial infarction. $\mathrm{Am} \mathfrak{F} \mathrm{Med}$ 1983;75:217-24

35 Chipkin SR, Frid D, Alpert JS, Baker SP, Dalen JE Aronin N. Frequency of painless myocardial ischaemia during exercise tolerance testing in patients with and
without diabetes mellitus. Am $\mathcal{F}$ Cardiol 1987;59:61-5.

36 Havlik RJ, Garrison RJ, Fabsitz R, Feinleib M. Variability of heart rate, P-R, QRS and Q-T duration in twins. $f$ Electrocardiol 1980;13:45-8.

37 Fagard R, Van Den Broeke C, Bielen E, Amery A. Maximum oxygen uptake and cardiac size and function in twins. Am f Cardiol 1987;60:1362-7.

38 Hanson B, Tuna N, Bouchard T, Heston L, Eckert E, Lykken D T, et al. Genetic factors in the electrocardiogram and heart rate of twins reared apart and together. gram and heart rate of twins

39 Wise BN, Durham NC, Comeau WJ, White PD. An electrocardiographic study of twins. Am Heart $\mathcal{F} 1939 ; 17$ : 701-10.

40 Schwartz PJ, Wolf S. QT interval prolongation as predictor of sudden death in patients with myocardial infarction. Circulation 1978;57:1074-7.

41 Haynes RE, Hallsrom AP, Cobb LA. Repolarisation abnormalities in survivors of out-of-hospital ventricular fibrillation. Circulation 1978;57:658-8.

42 Kahn JK, Sison JC, Vinik AI. QTc interval prolongation and sudden cardiac death in diabetic autonomic neuropathy. I Clin Endocrinol Metab 1987;64:751-4.

43 Bellavere F, Ferri M, Guarini L, Bax G, Piccoli A Prolonged QT interval in diabetic autonomic neuropathy: a possible role in sudden cardiac death? Br Heart 1988;59:379-83.

44 Ewing DJ, Boland O, Neilson JMM, Cho CG, Clarke BF Autonomic neuropathy, QT interval lengthening, and unexpected deaths in male diabetic patients. Diabetologia 1991;34:182-5.

45 Moller $\mathrm{P}$, Heiberg A, Berg $\mathrm{K}$. The atrioventricular conduction time-a heritable trait. Clin Genet 1982;21: 181-3.

46 Bielen E, Fagard A, Amery A. The inheritance of left ventricular structure and function assessed by imaging and Doppler echocardiography. Am Heart f 1991;121: 1743-9. 